\title{
DEBILIDAD DE LA VOLUNTAD Y AUTOCONTROL: UNA DISCUSIÓN EN TORNO A LA TEORÍA DE LAS FUNCIONES EJECUTIVAS
}

\section{THE WEAKNESS OF WILL AND SELF-CONTROL: A DISCUSSION AROUND THE THEORY OF EXECUTIVE FUNCTIONS}

\author{
Diego Landinez Guio* \\ Universidad Nacional de Colombia - Corporación Universitaria Minuto de Dios, CRS, \\ Bogotá - Colombia. \\ Recibido noviembre de 2018/Received November, 2018 \\ Aceptado julio de 2019/Accepted July, 2019
}

\begin{abstract}
RESUMEN
El presente artículo pretende reflexionar en torno a los conceptos de debilidad de la voluntad y del autocontrol desde la perspectiva de las Funciones Ejecutivas. Al situar el problema de la debilidad de la voluntad en el campo de las fallas en el autocontrol es posible examinar aspectos psicológicos y ambientales que intervienen en dicho fenómeno y tratar de entender cuáles son los aspectos generales que le dan origen. En este sentido, se quieren poner en discusión las tesis psicológicas de las Funciones Ejecutivas a partir de la idea de la voluntad extendida para examinar su viabilidad y sus limitaciones.

Palabras Clave: Debilidad de la voluntad, autocontrol, funciones ejecutivas, autorregulación, mejor juicio.
\end{abstract}

\begin{abstract}
This article aims to reflect on the concepts of the weakness of will and self-control from the perspective of the Executive Functions. By locating the problem of the weakness of the will in the field of failures in self-control, it is plausible to examine psychological and environmental viewpoints that intervene in in said phenomenon and try to understand which are the general aspects that introduce. In this sense, it is required to discuss the thesis of the Executive Functions from the idea of the extended willpower to examine its viability and its limitations.
\end{abstract}

Key Words: Weakness of the will, Self-control, Executive functions, Self-regulation, Better judgment.

\section{INTRODUCCIÓN}

En una primera aproximación, el concepto de debilidad de la voluntad — asociado a las discusiones en torno a la akrasía - podría definirse como la incapacidad de un individuo para tomar decisiones conforme a su mejor juicio (Mele, 2010), o como la imposibilidad de llevar a cabo una resolución ya tomada por la revisión de las intenciones (May \& Holton, 2012). Sin embargo, para Doucet y Turri (2014) este consenso mínimo está mediado por una polémica entre Holton y Mele que dista de haber agotado el tema, ya que los interrogantes en torno a los factores psicológicos

* Autor correspondiente / Corresponding author: dalandinezg@ hotmail.com 
y ambientales que intervienen en dicho fenómeno siguen abiertos y generan todavía diferentes respuestas e interpretaciones.

A partir de algunos experimentos, Doucet y Turri (2014) intentan mostrar que la atribución corriente de debilidad de la voluntad es sensible a elementos no psicológicos, como la capacidad para reconocer y alcanzar objetivos de largo alcance. Si bien el incumplimiento de compromisos evaluativos y prácticos (el mejor juicio y las intenciones) permite reconocer debilidad de la voluntad, los autores hallaron que en algunos casos tal condición no es absolutamente necesaria. Tanto la asociación con conductas estereotípicas como la naturaleza negativa de las consecuencias de una determinada acción están involucradas en el concepto y la atribución ordinaria de debilidad de la voluntad. Estos resultados permiten concluir a los autores que el fenómeno examinado incluye más variables que la mera revisión (irracional) de juicios e intenciones y que "el concepto popular de debilidad de la voluntad implica una falla en el ejercicio adecuado del autocontrol" (Doucet \& Turri, 2014, p. 3951, [traducción propia]).

Ubicar la debilidad de la voluntad en el marco conceptual del autocontrol permite pensar el problema de su atribución desde la perspectiva de los mecanismos psíquicos y sociales que permiten a los individuos establecer objetivos y trazar los posibles cursos de acción para alcanzarlos. Ahora bien, el concepto de autocontrol puede ser visto al menos desde dos enfoques generales: uno interno, que apela a procesos psíquico-somáticos; y otro externo, que recurre a factores ambientales. Por supuesto, cada uno de ellos dista de ser homogéneo o de tener conclusiones idénticas, por lo que podrían establecerse algunos puentes conceptuales, para que den cuenta, de una manera más completa, del funcionamiento del fenómeno estudiado y de dónde proceden sus fallas. En este sentido, la debilidad de la voluntad, entendida como un problema de autocontrol, puede ser explicada por el conjunto de mecanismos psíquicos que intervienen en el proceso de toma de decisiones, más que por una evaluación irracional de las intenciones (Bromhall, 2018; Haas, 2018); por la disposición ambiental sobre la cual actúa la voluntad (Heath \& Anderson, 2010); o por la inadecuación de los medios necesarios para lograr el autocontrol (Vierkant, 2014).

En el presente artículo, queremos explorar las posibilidades teóricas del modelo psicológico de las Funciones Ejecutivas para explicar el autocontrol y la debilidad de la voluntad, ya que parece mostrar el conjunto de procesos psíquicos que configuran la capacidad reflexiva de los seres humanos, considera sus limitaciones y plantean la posibilidad de su mejoramiento. En este orden de ideas, el texto se dividirá en tres partes: en la primera se expondrán los conceptos fundamentales de Funciones Ejecutivas y cómo puede entenderse la autorregulación y el autocontrol; en la segunda se examinarán, desde la perspectiva expuesta, el problema de los fallos en el autocontrol, el concepto de debilidad de la voluntad y cómo se puede fortalecer; en la tercera, se considerará el problema de los factores externos y de qué manera pueden o no ser compatibles con en el modelo de las Funciones Ejecutivas. En esta misma sección se expondrán algunos puntos de discusión crítica que señalan las principales limitaciones de este modelo explicativo.

\section{FUNCIONES EJECUTIVAS, AUTORREGULACIÓN Y AUTOCONTROL}

Las Funciones Ejecutivas (FEs) son un conjunto de procesos mentales de control que se requieren para estructurar tareas que demandan atención y concentración. Este conjunto de habilidades cognitivas configura el razonamiento, la capacidad para solucionar problemas, la planificación y la proyección, elementos esenciales para el desarrollo psicológico y social de los individuos, puesto que mejoran su capacidad para hacer frente, con cierto grado de éxito, a los retos que se presentan en diferentes entornos (Tirapu, Muñoz \& Pelegrín, 2002; Diamond, 2013). De acuerdo con la conceptualización de Adele Diamond (2013), existen tres tipos de FEs: el control inhibitorio (CI), la memoria de trabajo (MT) y la flexibilidad cognitiva (FC). De manera aislada o combinada, estas funciones abarcan los diferentes procesos señalados.

La inhibición o CI consiste en la capacidad de ejercer control sobre la atención, la conducta, los pensamientos y emociones, con el fin de superar las predisposiciones internas y distractores externos que impiden realizar una determinada tarea. Sin este tipo de control, no sería posible escapar del impulso de hábitos establecidos o de estímulos que determinarían eventualmente una determinada forma de actuar o una elección. Esta 
función permite ponderar las opciones respecto a qué hacer, cómo reaccionar y qué elegir en determinados casos, resistiendo al impulso ciego de factores internos y externos (Diamond, 2013). El CI de la atención o control de interferencia a nivel de la percepción permite enfocar a los individuos en aquello que eligieron y dejar de lado cualquier estímulo diferente; esta es una atención selectiva exógena que se enfoca en un estímulo sobresaliente. También hay un tipo de atención endógena que, inversamente, ignora voluntariamente un determinado estímulo para enfocarse en otros de acuerdo a los objetivos que se persigan.

La memoria de trabajo (MT) es un mecanismo por el cual se puede discurrir mentalmente en torno a determinados datos que no están presentes en la percepción inmediata o reciente. Esta función es importante para relacionar información adquirida en momentos diferentes y darle sentido, para incorporarla y actualizarla, para realizar operaciones matemáticas, llevar a cabo instrucciones, etc. Sin ella sería imposible el razonamiento y los procesos cognitivos que lo aparejan, como la capacidad de análisis y síntesis, la búsqueda de relaciones entre objetos y la creatividad. De igual manera, es la encargada de procesar el conocimiento conceptual (no solamente perceptivo), así como de influir en la toma de decisiones, el examen del pasado y la proyección futura (Tirapu et al., 2002; Diamond, 2013). Existen notables diferencias entre la MT y la memoria de corto plazo. Si bien en ambos casos se dispone de información, solo en la primera se opera con ella. A nivel cerebral, las conexiones también son distintas, sobre todo en lo que tiene que ver con la dependencia con respecto a la corteza dorsolateral prefrontal.

La MT no se halla aislada, sino que entra en relación con el CI. En algunos casos la MT procura el mejoramiento de los procesos de inhibición, específicamente cuando es necesario establecer la diferencia entre lo relevante y lo que no lo es. La MT interviene para disponer de la información y establece relaciones encaminadas al control de comportamientos y actitudes. De igual manera, la inhibición es necesaria para el funcionamiento de la MT, dado que para establecer relaciones entre ideas y dar paso a la novedad y la creatividad es indispensable establecer criterios de pertinencia, enfocar la atención en determinada información y dejar de lado otra. No obstante, cada una de estas FEs tiene su propio dominio y pueden actuar con cierta independencia, puesto que hay tareas que demandan atención, pero poco manejo de información, así como otras que, inversamente, requieren de un mayor dominio de datos, pero poca atención o inhibición de respuesta (Diamond, 2013).

La flexibilidad cognitiva (FC) es la capacidad de cambiar y adoptar puntos de vista, con respecto a los que se habían asumido previamente; para ello, se requiere de la acción de las otras dos FEs. Un cambio de perspectiva implica discurrir por la MT en la búsqueda de nuevas miradas e inhibir la propia. Es una FE que se enfoca en la asimilación y la adaptación a los cambios en el examen reflexivo de prioridades y posibilidades que pueden aparejar algún tipo de ventaja. En el campo de la FC se encuentra la creatividad, la capacidad para cambiar de tareas y la movilidad de la atención (Diamond, 2013).

De acuerdo con Hofmann, Schmeichel y Baddeley (2012), una perspectiva que integre las investigaciones de la psicología cognitiva y la psicología social es capaz de establecer vasos comunicantes entre las FEs, la autorregulación y el autocontrol. La autorregulación es un tipo de comportamiento dirigido a partir de objetivos temporalmente planificados; por otra parte, el autocontrol es el conjunto de procesos que pretenden reducir o anular la influencia de impulsos y estímulos no queridos, en tanto que su influencia impide lograr una determinada meta (Hofmann, Schmeichel, \& Baddeley, 2012). La propuesta sugiere que cada una de las FEs actúan directamente sobre diferentes aspectos de la autorregulación y que, por consiguiente, una falla en aquellas aumenta los niveles de riesgo sobre esta; de igual manera, si las primeras pueden entrenarse, la segunda también. Esta relación es posible por las condiciones que permiten el ejercicio exitoso de la autorregulación: i. la existencia de patrones que regulen el pensamiento, los sentimientos y los comportamientos; ii. motivación suficiente para disminuir la discrepancia entre dichos patrones y los estados efectivos; y iii. la capacidad suficiente para mantener una estrecha relación entre el patrón y los comportamientos, dada la visualización de los obstáculos de diversa índole que se interponen entre ellos (Hofmann et al., 2012; Baumeister \& Heatherton, 1996).

La MT permite establecer una representación clara y relevante de objetivos y del campo de acción que es necesario seguir para lograrlos. 
La inhibición es la encargada de rechazar aquellos estímulos externos e internos (distracciones y hábitos, por ejemplo) que impiden enfocar la atención en los objetivos trazados y que se interponen en su prosecución. La FC está a la base de la reevaluación de fines y medios, pues con ella se mejora la adaptabilidad de los objetivos a los medios disponibles y la creatividad en la elección de los medios, una vez se determine su mayor o menor capacidad para orientar los diferentes cursos de acción posibles. De acuerdo con esta síntesis, cada FE está involucrada en aspectos que actúan juntos en la autorregulación, ya que subyacen a la capacidad para planear, diseñar y reestructurar diferentes metas y sus posibles direccionamientos (Hofmann et al., 2012).

En lo que respecta al autocontrol, cuya función implica resistir la tentación de actuar o reaccionar con base en algún impulso no ponderado correctamente, es evidente el papel de las FEs, pues ellas están involucradas en el desarrollo de la disciplina necesaria para realizar y completar una actividad sin interferencia de ninguna distracción, por más atrayente que sea. En particular, el CI aunado a la MT están involucrados en la "postergación de la satisfacción" (delay gratification) que se requiere para la concentración en una tarea específica y la subordinación de los cursos de acción necesarios para llegar a ella, porque sin el ejercicio conjunto tales FEs sería imposible enfocar la atención en objetivos de cierto grado de complejidad, como escribir una ponencia o realizar un negocio (Diamond, 2013).

\section{FALLAS DE AUTOCONTROL Y DEBILIDAD DE LA VOLUNTAD}

El modelo de las FEs complementa la perspectiva del "sistema dual" del comportamiento humano que distingue los impulsos y el autocontrol (Hofmann, Friese, \& Strack, 2009). Los impulsos son aquellas respuestas específicas e inmediatas que se activan en función de estímulos ambientales, en condiciones espacio-temporales determinadas. Un comportamiento impulsivo está determinado por la inmediatez de la apetencia y choca "con el logro de objetivos a largo plazo o genera conflictos interpersonales en el mismo sentido", de ahí que su contraparte, el autocontrol, "definido aquí como la capacidad para anular o inhibir 'tendencias de comportamiento indeseadas (...) y abstenerse de actuar conforme a ellas"”, sea "una habilidad importante para el funcionamiento diario" (Hofmann et al., 2009, p. 163, [traducción propia]), dado que permite alcanzar los objetivos trazados, limitando el alcance de las distracciones que puedan interponerse entre la adecuación entre medios y fines de la acción.

En términos generales, se puede decir que el sistema impulsivo o pulsional prepara al organismo para llevar a cabo funciones básicas de supervivencia, con base en asociaciones y representaciones entre las necesidades vitales y los estímulos ambientales. En contraste, el sistema reflexivo funciona con operaciones mentales de alto nivel para regular objetivos de largo plazo y facilitar la toma de decisiones, en cuanto forma una especie de muro de contención frente a los impulsos más inmediatos. Por consiguiente, este sistema dual toma la forma de un combate entre dos mecanismos contrapuestos, aunque complementarios, que se enfrentan en torno a los posibles cursos de acción de los individuos (Hofmann et al., 2009).

El autocontrol afronta de manera permanente la influencia de factores internos y externos que impiden su ejercicio apropiado, en circunstancias en las cuales el sistema reflexivo pierde el direccionamiento de las decisiones. ¿Cuáles son las causas de este fenómeno? Siguiendo a Hofmann et al. (2009), el funcionamiento del sistema dual está determinado por una serie de situaciones y disposiciones que están en condiciones de inclinar la balanza de uno u otro lado y predecir si el comportamiento de una persona puede, en tales casos, ser más reflexivo que impulsivo o viceversa. En efecto, hay circunstancias como el agotamiento del ego, la presión social, la intoxicación, entre otras, que ponen en riesgo la capacidad reflexiva de los individuos (Hofmann et al., 2012; Baumeister \& Heatherton, 1996). De acuerdo con Diamond, las FEs no son invulnerables ante influencia de las diferentes variables que afectan la vida de los seres humanos. Los problemas afectivos, psíquicos y somáticos como el estrés, la falta de sueño o el deterioro en la salud física y mental desencadenan falencias en la capacidad para razonar, resolver problemas y la autodisciplina (Diamond, 2013). Por consiguiente, las fallas en el autocontrol se deben, desde estas perspectivas, tanto a la fuerza que muestren los impulsos para sobrepasar la capacidad reflexiva, como a los factores que debiliten las FEs. 
¿Cómo podría caracterizarse la debilidad de la voluntad desde esta perspectiva? $\mathrm{Si}$, como concluyen Doucet y Turri (2014), la debilidad de la voluntad no es solamente una revisión de compromisos prácticos, sino además un problema "para formar compromisos que son requeridos por los propios objetivos a largo plazo", que "implica un fallo para reconocer lo que sería mejor hacer a la luz de los propios objetivos generales" (p. 3952, [traducción propia]), se puede vislumbrar una dificultad en la MT, pues el error estaría en la capacidad reflexiva para visualizar con claridad tanto los objetivos como los cursos de acción más adecuados para alcanzarlos.

Monika Betzler (2009) señala también que una persona es débil de voluntad cuando presenta una incoherencia entre las acciones y las "intenciones razonables", cuyos objetivos no logran coordinar para sí, a lo largo del tiempo, ni la cantidad ni la calidad de medios necesarios para su consecución, llegando a un eventual cambio de objetivos o a un estado de indeterminación, en el que "no es ni falso ni verdadero que los medios que ella omite debilitan el fin proyectado" (pp. 213-4), teniendo la capacidad para procurarlos de manera efectiva. En este sentido, la debilidad de la voluntad plantea un problema de conexión entre fines y medios que puede ser explicado desde el punto de vista del CI, que radicaría en una incapacidad para distinguir los mejores objetivos de aquellos que no lo son tanto y de eludir aquellos que, si bien pueden ser más placenteros o llamativos, no son los más adecuados a largo plazo, al igual que los medios. De igual manera, alguien con debilidad de la voluntad puede fijar representaciones y no ser capaz de crear nuevos objetivos a largo plazo (problemas de FC), más adecuados a un "estándar de racionalidad objetiva" (Betzler, 2009, p. 193) ${ }^{1}$. Este tipo de fallas en las FEs no solo impedirían la formación de los mejores juicios, sino también de inhibir los cursos de acción que se desvían de ellos.

Ahora bien, si la debilidad de la voluntad descansa sobre fallos en el autocontrol y en las FEs que lo sustentan, también está sujeta al influjo de factores como la salud física y emocional de los individuos, las presiones del ambiente, etc., pero, además, debe tener la capacidad de ser controlada con el mejoramiento de lo que anteriormente se llamó el sistema reflexivo. De hecho, varios autores ya citados (Baumeister \&
Heatherton, 1996; Hofmann et al., 2009, 2012; Diamond, 2013), proponen la posibilidad de tal mejoramiento y también hay evidencia empírica que parece sustentar sus hipótesis.

Mark Muraven (2010) sostiene que el autocontrol se puede mejorar con entrenamiento continuo a partir de tareas pequeñas, enfocadas en el fortalecimiento de la capacidad para inhibir estímulos perturbadores. Para probar esta hipótesis, llevó a cabo un experimento con 92 personas de ambos sexos en el que se sometieron a dos semanas de entrenamiento de su autocontrol; con tal fin, fueron distribuidas al azar en cuatro grupos con una tarea determinada que debían realizar y registrar diariamente: evitar el consumo de dulces, ejercitarse con una manopla, elaborar operaciones matemáticas o llevar un diario. De acuerdo con el autor, las dos primeras tareas requieren de un esfuerzo para inhibir, por un lado, la tentación de consumir dulces y, por otro, la tendencia a no realizar un ejercicio físico, mientras que las otras dos no lo demandan. Antes y después del experimento, los voluntarios fueron sometidos a la tarea de la señal de pare (stop signal task) para medir en cada caso su capacidad inhibitoria. El resultado general mostró que luego de las dos semanas de realizar cada una de las tareas, los dos primeros grupos mejoraron su desempeño en el control inhibitorio, mientras que los otros no lo hicieron, pese a que quienes realizaron la tarea del diario consideraban que su esfuerzo era similar al de los primeros.

Un estudio de Moffitt, Arseneault, Belsky, Dickson, Hancox, et al. (2011), llevó un seguimiento de 32 años a 1.000 niños nacidos entre 1972 y 1973 en Dunedin, Nueva Zelanda, con una tasa de permanencia de $96 \%$. La naturaleza del estudio era observacional y correlacional, es decir, no intervenía en la dinámica propia de los sujetos estudiados. La hipótesis que se comprobó es que los niños con mayores índices de autocontrol obtienen, eventualmente, mejores resultados académicos, laborales y económicos, son menos propensos a hacerse dependientes de las drogas y el alcohol o a sufrir mayores problemas de salud. Se estableció que los niños entre 3 y 11 años con mejor control inhibitorio (CI) —es decir, aquellos que se distraían menos, que eran más persistentes y menos impulsivos- eran más propensos en la adolescencia a permanecer en la escuela y estaban menos expuestos a consumir drogas, a fumar o a ser padres a temprana edad. De la misma manera, 
crecían con mejor salud, ganaban más dinero y eran "mejores ciudadanos" al cumplir los 30 años que aquellos que presentaban un menor CI de niños.

El primer estudio citado muestra que es posible mejorar el autocontrol con base en ejercicios que fortalecen las FEs, en este caso el CI. El segundo, establece que, a mayores índices de autocontrol, mejores condiciones de vida para los individuos. Aunque no pueden considerarse conclusiones definitivas, dado que sería necesario tomar en cuenta un mayor número de variables (como las demás FEs, diferentes factores ambientales, etc.), sí sugieren que un mejoramiento en la práctica de las FEs redunda en una mayor fuerza en el autocontrol, que quienes lo logran se autorregulan mucho mejor y, por consiguiente, son menos propensos a ser considerados débiles de voluntad, pues son capaces de trazar mejores objetivos a largo plazo y elegir los cursos de acción más correctos para alcanzarlos.

No obstante, esta lectura de la debilidad de la voluntad y el autocontrol se puede problematizar en, al menos, dos elementos. El primero de ellos es el carácter jerárquico de las funciones mentales, que supone en el sistema evaluativo una capacidad esencial de dirigir los impulsos y los hábitos en el comportamiento humano; el segundo, es la irracionalidad que se le atribuye al desfase entre las acciones y los juicios. Desde la perspectiva de William James, Kyle Bromhall (2018) toma distancia de las concepciones filosóficas clásicas de la debilidad de la voluntad, al igual que la versión cristiana del fenómeno, para mostrar que las acciones que van en contravía del mejor juicio no se deben a un problema de racionalidad ni de moralidad, sino de la existencia de hábitos arraigados que se activan automáticamente ante determinadas situaciones en las que se evalúa como mejor otro curso de acción, pero que carece de fuerza suficiente frente a las respuestas que ya están establecidas.

La formación de los hábitos permite a los individuos un ahorro energético en la realización de las acciones, de modo que las respuestas automáticas a condiciones habituales de comportamiento se revelan como placenteras frente a modificaciones abruptas en los cursos de acción (Bromhall, 2018). Esto implica que la competencia entre acciones posibles ante estímulos y condiciones ambientales determinadas está mediada por la formación de hábitos, propia de la configuración del comportamiento humano, y no solo por la evaluación racional de los cursos de acción, dado que los primeros tienen una "fuerza motivacional" más fuerte que los segundos y, por ello, son más placenteros (Bromhall, 2018, p. 36). Si las acciones están guiadas por el hábito, el agente no requiere de esfuerzo para llevarlas a cabo. Solo el encuentro con objetos que demandan ir en contra del hábito o que demandan respuestas no habituales, hacen transparente el esfuerzo de la acción por el displacer que generan.

En tal caso, una acción no habitual requiere de un mayor esfuerzo del agente para ser realizada, ya que la motivación racional del mejor juicio debe competir con otras no racionales como los sentimientos y los deseos. En este problema, que hace consciente el esfuerzo requerido para la acción, se da la debilidad de la voluntad como un conflicto de motivaciones, que resulta "de una falta de concentración debido a la fatiga, a ideas conflictivas o, incluso, a la falta de atención" (Bromhall, 2018, p. 39, [traducción propia]). La akrasia se entiende, en este sentido, como una especie de "letargo" de ciertas disposiciones psicológicas que impiden que un individuo actúe en contra de sus hábitos establecidos, en función de una evaluación racional de las posibles acciones que compiten ante una determinada situación.

La propuesta jamesiana de Bromhall (2018) no es incompatible con la de las FEs, pues reconoce la capacidad de ejercitar y mejorar la capacidad cognitiva para superar los patrones habituales de comportamiento, pero matiza el carácter práctico del fallo de la debilidad de la voluntad y reconoce el peso de motivaciones no racionales en las decisiones humanas. En esta última dirección argumental, Julia Haas (2018) propone entender la debilidad de la voluntad desde una teoría de la acción y la decisión que parte de una comprensión de la mente como un sistema múltiple y no jerárquico. Para esta autora existen tres dimensiones del Modelo Multi-Sistema de la mente (Multi-System Model of the mind - MSM) que operan con relativa independencia, en la valoración de las acciones posibles para determinados estímulos. Existe un sistema "innato" (Hardwired), cuyas respuestas automáticas son apropiadas para problemas simples; uno deliberativo, que opera adecuadamente en ambientes complejos no familiares; y uno habitual, que responde de manera simplificada a situaciones conocidas de alto grado de complejidad 
(Haas, 2018). Así pues, para realizar una acción, un agente pone en funcionamiento los tres sistemas, según las necesidades del ambiente y las demandas de las tareas a realizar.

Cada subsistema del MSM proporciona un "perfil de precisión" respecto a la valoración de determinada acción de manera independiente y, en la comparación de todas las estimaciones, se selecciona el perfil más alto para coordinar el curso de acción conveniente, mas esta evaluación no depende del sistema deliberativo, sino de la operatividad misma del MSM. Desde esta teoría, dice Haas (2018), se comprende que

un agente puede juzgar que es mejor hacer A en lugar de B (en el sentido de que uno de sus sistemas de decisión valora A más que B) y aún hacer B, si ese sistema no es el asignado para la selección de las acciones. (p. 5184, [traducción propia]).

Una importante conclusión que se extrae de esta propuesta es que la debilidad de la voluntad es un "subproducto" de los procesos de decisión y no una irregularidad racional, es decir, que es "un resultado inevitable" del MSM, ya que "los sistemas múltiples de decisión operan en paralelo, intercambiándose para optimizar la toma de decisiones" (Haas, 2018, p. 5191, [traducción propia]). En este sentido, la debilidad de la voluntad se muestra como un problema práctico de optimización de las respuestas operativas del sistema, que no depende del gobierno del sistema deliberativo, sino de su relación con los demás, de ahí que para Haas (2018) esta explicación sea válida para la definición clásica de la debilidad de la voluntad (ir en contra del mejor juicio) más que para la que la asocia a los problemas del autocontrol (ir en contra de las intenciones).

\section{LOS FACTORES EXTERNOS: LA OBJECIÓN DE LA "VOLUNTAD EXTENDIDA"}

El concepto de autocontrol parece estar relacionado directamente con el bienestar de cada quién y de la responsabilidad que tiene consigo mismo. Sin embargo, el autocontrol y sus fallas también afectan la relación que un individuo tiene con los otros, pues, como lo sugiere Hofmann et al. (2009), el sistema impulsivo por sí solo se interpone en las relaciones interpersonales $\mathrm{y}$, como afirman Moffitt et al. (2011) al comienzo de su estudio:

La necesidad de postergar la gratificación, controlar los impulsos, y modular la expresión emocional es la más temprana y la más extendida demanda que la sociedad coloca en sus niños, (...) el éxito en las tareas de la vida depende críticamente del dominio que tengan de tal autocontrol. (p. 2693, [traducción propia]).

Si ello es así, la importancia del autocontrol no recae únicamente en lo que el individuo determina para sí mismo, sino en la capacidad que tiene para alcanzar objetivos establecidos por la sociedad a la que pertenece. De ahí que la atribución de debilidad de la voluntad tenga, al menos en su acepción más común, una referencia a objetivos y cursos de acción que se espera socialmente (o estereotípicamente, como dicen Doucet y Turri, 2014), que un agente persiga en determinadas circunstancias. Desde luego, esta observación no cuestiona la necesidad individual del autocontrol, sino que resalta la conexión entre las capacidades psicológicas individuales y el contexto en el que viven, en tanto que no solo es externa su demanda, al menos en un primer momento, sino que son externas también las herramientas que permiten adquirirlo y mejorarlo.

Si se adopta el modelo de las FEs y la capacidad que varios autores ven en mejorar su operatividad, no se puede obviar que tal educabilidad depende de los factores externos a los que sean sometidos los individuos: desde el modelo educativo en el que se encuentran para desarrollar tales capacidades, hasta el contexto familiar, las expectativas sociales, etc., que les demandan su uso. En este sentido, no es arriesgado suponer que, si la debilidad de la voluntad es una falla de autocontrol, debe tenerse en cuenta la influencia del ambiente en los sujetos a quienes se les atribuye, incluso asumiendo la perspectiva de las FEs, pues es el ejercicio continuo lo que permite desarrollar las capacidades de autocontrol y sería una abstracción pensar que sin las condiciones que demanden el uso de operaciones cognitivas de alto nivel, estas puedan desarrollarse con ejercicios aislados.

Ahora bien, una crítica fuerte al modelo psicológico dual proviene de la idea de la "voluntad extendida". De acuerdo con Heath y Anderson (2010), "lo que las teorías experimentales de los juegos pueden haber demostrado no es que la 
gente es sistemáticamente irracional, sino que la racionalidad humana está fuertemente andamiada (scaffolded). Removido el andamiaje, las cosas ya no pueden hacerse tan bien" (p. 233, [traducción propia]). Su propuesta pretende evidenciar que el ambiente hace parte esencial de los procesos cognitivos y volitivos, ya que los procesos evolutivos humanos no tienen que ver únicamente con una adaptación biológica, sino también con adaptaciones lingüísticas y sociales. Desde esta óptica, el problema de la "procrastinación", en tanto problema de dispersión de la atención, no se resuelve en función de un mejoramiento de habilidades cognitivas, sino en el mejoramiento del andamiaje del individuo con su ambiente.

Heath y Anderson (2010) consideran exagerado el peso que suele darse a la autodisciplina para solucionar problemas de autocontrol, pues, por un lado, el concepto de autodisciplina adolece de comprobaciones empíricas y, por otro lado, varias investigaciones han mostrado que "aunque la fuerza de voluntad es como un músculo, y puede ser entrenada, también puede llegar a agotarse" (p. 245, [traducción propia]). Por lo tanto, las estrategias de autocontrol que proponen están encaminadas a asumir la estrecha relación de los seres humanos con su ambiente y que sea ella la que permita bloquear los estímulos que genera la procrastinación.

No vamos a detenernos en los aspectos específicos de la propuesta de Heath y Anderson, puesto que nos interesa su perspectiva crítica, pero sí queremos plantear algunos problemas que quizá genere el enfrentamiento entre estos dos modelos, el psicológico de las FEs y el de la voluntad extendida. En primer lugar, la utilización del medio como barrera para los estímulos que provocan problemas de autocontrol no parece otra cosa que una extensión del CI, de la misma manera que la organización del ambiente un ejercicio de la MT. La disposición de los espacios físicos de trabajo se puede revelar como un mecanismo complejo de reflexión en el que interactúan dinámicamente las FEs y el ambiente circundante. En este sentido, y si puede establecerse dicha relación, ¿no sería necesario que, partiendo de las disposiciones del espacio, se logre mejorar las FEs? Esto permitiría plantear una relación más estrecha entre el individuo y su ambiente, en la medida en que se logre evidenciar cómo las FEs modifican y son modificadas, a su vez, por los contextos en los que se halla inmerso un individuo.

Por otro lado, si se aplican condicionamientos externos, ¿esto puede considerarse realmente autocontrol? O, en todo caso, ¿no supone la capacidad cognitiva para establecer los objetivos correctos y los cursos de acción necesarios para diseñar el espacio sobre el que se plasman las estrategias de autocontrol? ¿Son suficientes tales herramientas externas si no se desarrolla la capacidad para inhibir ciertos impulsos o para enfocar la atención en determinados objetivos? Estas preguntas sugieren la necesidad de encontrar los ejes que permitan anclar las FEs en las diversas maneras en que los individuos se relacionan con el mundo, pues parece que es en tales puntos de contacto que se tejen las estrategias para solucionar los problemas de autocontrol.

Si la debilidad de la voluntad se entiende como un fallo en el autocontrol que deliberadamente se comete - pues sin el factor de la libre elección no se daría realmente-, ¿puede entenderse el autocontrol en función exclusiva de una coacción exterior? Como en el ejemplo del aprendizaje de ciertas operaciones matemáticas con el ábaco, en el que llegado a un punto no es necesario, pues se ha interiorizado su función (pese a los vestigios visibles en el movimiento de los dedos), ¿no sería necesario que se interiorizaran las estrategias de autocontrol para que no se dependa únicamente de condiciones externas en el caso de que ellas falten? ¿Quiere decir esto que en ausencia de ciertos factores externos es imposible el autocontrol?

Tillmann Vierkant (2014) afirma, siguiendo a Fabio Paglieri, que la propuesta teórica de Heath y Anderson no distingue entre la fuerza de voluntad y los recursos externos que se disponen para mejorar el autocontrol, por lo que asumen los dos procesos como uno solo. La tesis de Vierkant, en este sentido, es que los recursos psíquicos que configuran la fuerza de voluntad y el uso de ayudas externas son dos mecanismos que se utilizan como medio para lograr el autocontrol: "el proceso es el mismo, pero los sistemas que se coordinan para llevarlo a cabo son distintos, con funciones y economías cognitivas propias" (Vierkant, 2014, p. 58, [traducción propia]). Con este matiz, se muestra la complementariedad entre los mecanismos psíquicos y la disposición del medio para lograr el autocontrol, sin confundir sus respectivas especificidades. 
Vierkant (2014) se sirve de la distinción entre control evaluativo y control directivo para mostrar cómo los procesos de formar intenciones (control evaluativo) es un proceso no intencional, en tanto que hace parte del funcionamiento del sistema global de toma de decisiones (control directivo). Ello implica que el autocontrol no es un proceso necesariamente consiente, ni dependiente del sistema deliberativo (en términos de Haas, 2018), sino que se puede potenciar con la evaluación (consiente) de las acciones posibles y la formación del mejor juicio. En este sentido, tanto la fuerza de voluntad como la manipulación del ambiente para controlar impulsos no deseados, afrontan desafíos distintos, en tanto que la primera se enfoca en potenciar procesos intencionales y la segunda en actuar sobre aquellos que no lo son, en función de una misma finalidad: mejorar el control directivo.

\section{CONCLUSIONES}

El examen de las FEs permite explorar algunos aspectos operativos del sistema de toma de decisiones y cómo se gesta, en medio de ellos, la debilidad de la voluntad en las complejas dinámicas del autocontrol. Sin embargo, el problema de actuar en contra del mejor juicio, así como las posibilidades para mejorar los procesos de control directivo de la mente, se comprenden mejor si se toma en cuenta la multiplicidad del sistema. La debilidad de la voluntad es, entonces, el resultado del proceso mismo de toma decisiones (Haas, 2018), que no se gesta en la irracionalidad del agente, sino de la interferencia de los procesos relativamente autónomos que componen la estructura psicológica de los individuos, entre ellos la existencia de hábitos, cuya función es la economía energética en la realización de acciones (Bromhall, 2018).

Ahora bien, aunque la revisión de las FEs y su papel en el comportamiento humano revela aspectos importantes en el control de las acciones, como la disposición de objetivos y medios, la inhibición de factores internos y externos perturbadores, así como la capacidad de cambiar los objetos de la atención (Diamond, 2013), esta postura desestima la importancia de la modificación del ambiente en el logro del autocontrol y, en cambio, pone el mayor peso de dicho proceso en la fortaleza de las FEs y su capacidad para ser entrenadas (Baumeister \& Heatherton, 1996; Hofmann et al., 2009; Muraven, 2010; Hofmann et al., 2012; Diamond, 2013). Por otro lado, Heath y Anderson (2010) enfatizaron en la relación necesaria entre la voluntad y la modificación del ambiente, a tal punto que pusieron el acento en los factores ambientales más que en la operatividad del sistema mental de la toma de decisiones, lo que condujo a considerar una serie de cuestionamientos sobre la relación entre los factores psicológicos y ambientales que intervienen en la realización de las acciones.

La ventaja de las reflexiones de Vierkant (2014) es que establece las diferencias entre la fuerza de voluntad y el recurso a las modificaciones del entorno y su raíz común, en tanto que son medios distintos para mejorar los procesos de autocontrol. De ello se puede concluir que la toma de decisiones y, por consiguiente, la debilidad de la voluntad, se encuentran arraigadas en un sistema complejo de interacción entre el sistema múltiple de la mente, cuyas FEs solo son constitutivas del sistema deliberativo, y las condiciones ambientales, que determinan a la vez que tienen la posibilidad de ser determinados por la acción humana. Esto implica que no hay incompatibilidad entre la teoría de las FEs y la voluntad extendida, sino que cada proceso que fortalece tanto la fuerza de voluntad como la modificación del medio opera desde y sobre un aspecto distinto del sistema de la mente, y contribuye de manera relativamente autónoma a un mejoramiento del autocontrol. 


\section{Referencias}

Baumeister, R. \& Heatherton, T. (1996). Self-Regulation Failure: An Overview. Psychological Inquiry, 7(1), 1-15.

Betzler, M. (2009). Debilidad de la voluntad como irracionalidad furtiva. Ideas y Valores, 58(141), 191-215.

Bromhall, K. (2018). Embodied Akrasia: James on Motivation and Weakness of Will. William James Studies, 14(1), 26-53.

Diamond, A. (2013). Executive Functions. Annual Review of Psychology, 64, 135-68. doi: 10.1146/ annurev-psych-113011-143750

Doucet, M. \& Turri, J. (2014). Non-psychological Weakness of Will: self-control, stereotipes, and consequences. Synthese, 191(16), 13935-3954. doi: 10.1007/s11229-014-0508-0

Haas, J. (2018). An empirical solution to the puzzle of weakness of will. Synthese, 195(12), 5175-5195. doi: https://doi. org/10.1007/s11229-018-1712-0

Heath, J. \& Anderson, J. (2010). Procrastination and the Extended Will. En C. Andreou \& M. White (Eds.), The Thief of Time: Philosophical Essays on Procrastination (pp. 233-253). New York: Oxford University Press.

Hofmann, W., Friese, M., \& Strack, F. (2009). Impulse and Self-Control from a Dual-Systems Perspective. Perspectives on Psychological Science, 4(2), 162-176.
Hofmann, W., Schmeichel, B., \& Baddeley, A. (2012). Executive functions and self-regulation. Trends in Cognitive Sciences, 16(3), 174-180.

May, J. \& Holton, R. (2012). What in the world is weakness of will? Philosophical Studies: An International Journal for Philosophy in the Analytic Tradition, 157(3), 341-360.

Mele, A. (2010). Weakness of will and akrasia. Philosophical Studies: An International Journal for Philosophy in the Analytic, 150(3), 391-404.

Moffitt T., Arseneault L., Belsky D., Dickson N., Hancox R., et al. (2011). A gradient of childhood self-control predicts health, wealth, and public safety. PNAS, 108(7), 2693-2698

Muraven, M. (2010). Building Self-Control Strength: Practicing Self-Control Leads to Improved Self-Control Performance. $J$. Exp. Soc. Psychol., 46(2), 465-468.

Tirapu, J., Muñoz, J. M., \& Pelegrín, C. (2002). Funciones ejecutivas: necesidad de una integración conceptual. Revista de Neurología, 34(7), 673-685.

Vierkant, T. (2014). Mental Muscles and the Extended Will. Topoi, 33(1), 57-65. 


\section{Notas al final}

1

En la medida en que la concepción clásica [de la debilidad de la voluntad] describe, sin embargo, un conflicto sincrónico entre los juicios acerca de lo mejor y los deseos está sugiriendo una concepción de la voluntad débil como un genitivo subjetivo. De esta puede distinguirse una concepción en términos de procesos diacrónicos relativa a la voluntad débil como un genitivo objetivo. Muchos casos de debilidad de la voluntad deben entenderse, en este último sentido, como acciones en contra de intenciones razonables y, consiguientemente, como formas de irracionalidad furtiva. (Betzler, 2009, p. 213). 